\section{Avaliação da Suscetibilidade do LDL de Pacientes Com Diabetes Mellitus Tipo 1 à Oxidação in vitro e Sua Relação Com o Controle Glicêmico}

\section{RESUMO}

Avaliamos em 38 pacientes com diabetes tipo 1 (DM1) e 24 não diabéticos, a suscetibilidade do LDL à oxidação in vitro. Foram avaliados glicemias de jejum e pós-prandial (GPP), hemoglobina glicosilada (HbAlc), perfil lipídico e análise espectrofotométrica da oxidação do LDL antes e 1, 3, 6 e 24 horas após adição de substância oxidante sulfato de cobre $\left(\mathrm{CuSO}_{4}\right)$. O coeficiente de oxidação do LDL foi semelhante nos dois grupos antes da adição do $\mathrm{CuSO}_{4}$. Entretanto, 3 horas após, o LDL se mostrou mais suscetível à oxidação in vitro nos pacientes com DM1. Houve correlação negativa com a GPP $(r=-0,2511 ; p<0,05)$ e com a HbAlc $(r=-0,2541 ; p<0,05)$. Concluímos que, em nossa amostra, o LDL dos pacientes com DM1 foi oxidado mais precocemente que o dos não diabéticos, e que o controle glicêmico apresentou importância neste evento. (Arq Bras Endocrinol Metab 2004;48/4:513-517)

Descritores: Diabetes tipo 1; LDL oxidado; Suscetibilidade; Controle glicêmico

\begin{abstract}
Evaluation of LDL Susceptibility of Patients With Type 1 Diabetes to in vitro Oxidation and Its Relation to Glycemic Control.

In 38 patients with type 1 diabetes (DM 1) and 24 non-diabetics we investigated LDL susceptibility to in vitro oxidation. Fast and post-prandial glycaemia (PPG), glycated hemoglobin (HbAlc) and lipid profile were determined, together with an spectrophotometric analysis of LDL oxidation before and 1, 3, 6 and 24 hours after addition of the oxidant substance - copper sulphate $\left(\mathrm{CuSO}_{4}\right)$. The LDL oxidation coefficient in the two groups presented similar basal values; however 3 hours after $\mathrm{CuSO}_{4}$, LDL was more oxidized in patients with DM1. There was a negative correlation with PPG $(r=-0.2511 ; p<0.05)$ and $\mathrm{HbAlc}(r=-0.2541 ; p<0.05)$. We conclude that in our sample of DM patients LDL was oxidized earlier than in the non-diabetics and that the glycemic control is important in this event. (Arq Bras Endocrinol Metab 2004;48/4:513-517)
\end{abstract}

Keywords: Type 1 diabetes; Oxidized LDL; Susceptibility; Glycemic control

O DiABETES MELlitus (DM) é a doença metabólica mais comum e apresenta sérias implicações na qualidade de vida dos pacientes em decorrência das suas complicações microvasculares e macrovasculares. Atualmente, esta enfermidade é a principal causa de cegueira e responsável por um considerável percentual de doença renal terminal nos Estados Unidos da América (1).

Um dos fatores determinantes de evolução para as complicações crônicas microvasculares é o controle glicêmico, conforme demonstrado pelo Diabetes Complications Control Trial (2). Neste estudo, entretanto, não foi artigo original

Simone H. de Castro

Hugo C. Castro-Faria-Neto

Eliete L.S. Clemente

Marilia B. Gomes

Disciplina de Diabetes e

Metabologia do Hospital

Universitário Pedro Ernesto,

Universidade do Estado do

Rio de Janeiro,

Rio de Janeiro, RJ. 
observada redução do risco de complicações macrovasculares com a terapia intensiva do diabetes. Esta avaliação possivelmente foi limitada pelo reduzido número de eventos cardiovasculares ocorridos durante o período do estudo.

A doença cardiovascular é a principal causa de morte em ambos os tipos de DM, perfazendo $44 \%$ do total de casos de morte nos pacientes com DM tipo 1 (DMl), seguida pela doença renal (2l\%) (3). Droga e cols. (4) mostraram que estes pacientes apresentam disfunção endotelial mesmo com níveis glicêmicos próximos da normalidade e taxa de excreção urinária de albumina normal, sugerindo que este fenômeno precede a microalbuminúria como um marcador de risco precoce para a doença cardiovascular. Em adição a estes dados, Järvisalo e cols. (5) demonstraram que mesmo crianças com DMl já estão sujeitas às complicações cardiovasculares da doença e apresentam espessamento do complexo íntima-média. Estes achados levantam a hipótese da existência de outros fatores envolvidos na fisiopatologia da aterosclerose no DMl, além da hiperglicemia.

O LDL oxidado tem sido caracterizado como um fator crítico no processo aterosclerótico tanto em modelos animais como em humanos (6). A modificação oxidativa do LDL confere propriedades específicas pró-aterogênicas a esta molécula, a qual tem ação na célula endotelial, nos macrófagos e células musculares lisas.

O presente trabalho tem como objetivo avaliar a suscetibilidade à oxidação in vitro do LDL de pacientes com DMl acompanhados regularmente no ambulatório de Diabetes do Hospital Universitário Pedro Ernesto.

\section{PACIENTES E MÉTODOS}

Foram recrutados 38 pacientes com DMl acompanhados regularmente no ambulatório de Diabetes do
Hospital Universitário Pedro Ernesto (HUPE) - UERJ, e 24 não diabéticos, pareados por sexo, idade e índice de massa corporal (IMC), após assinatura do termo de consentimento previamente aprovado pelo comitê de ética do HUPE. As características das duas amostras no momento da coleta dos exames estão na tabela 1 .

Os critérios de exclusão para aqueles com DM foram tabagismo, etilismo, presença de nefropatia diabética, infecção sistêmica e/ou uso de medicamentos que pudessem alterar os resultados da determinação da suscetibilidade do LDL à oxidação, como os inibidores da ECA, sulfato ferroso e complexos vitamínicos e de sais minerais contendo vitaminas $\mathrm{C}$ e $\mathrm{E}$ e $\mathrm{Zn}, \mathrm{Se}, \mathrm{Fe}$ e Cu. No grupo sem DM, estes critérios incluíam, também, a presença de familiares diretos portadores de DM.

Os participantes foram submetidos à coleta de sangue após 12 horas de jejum e 2 horas após café da manhã habitual. Os exames realizados foram glicemia de jeum (GJ) e pós-prandial (GPP) (glicose oxidase); colesterol total, HDL e triglicerídeos através de reações colorimétricas com leitura pelo aparelho Cobas-Mira (Roche); hemoglobina glicosilada (HbAlc - HPLC com leitura pelo aparelho Merck Hitachi 9100 - VR 2,6 a 6,2\%). O LDL foi calculado pela fórmula de Friedwald (7).

Os pacientes tiveram seu controle glicêmico classificado de acordo com os critérios descritos por Chase (divisão do valor da $\mathrm{HbAlc}$ do paciente pelo valor do limite superior da normalidade do método) da seguinte forma: bom controle (índice $<1,33$ ), controle regular (índice 1,33 e $<1,5$ ) e péssimo controle (índice 1,55) (8).

Para a análise da suscetibilidade do LDL à oxidação in vitro, foram coletados $20 \mathrm{~mL}$ de sangue a vácuo em tubos com EDTA, os quais foram centrifugados a $4^{\circ} \mathrm{C}$ por 20 minutos a $800 x g$. Após a separação do plasma, o mesmo foi imediatamente processado para o isolamento do LDL seguindo as etapas descritas: ajuste da densidade para $1,3 \mathrm{~g} / \mathrm{mL}$ pela

Tabela 1. Características do grupo de pacientes com DM1 e sem DM no momento da coleta dos exames.

\begin{tabular}{lccc}
\hline Variáveis & DM1 & Sem DM & p \\
\hline $\mathrm{N}$ & 38 & 24 & \\
Sexo (F/M) & $15 / 23$ & $14 / 10$ & - \\
Idade (anos) & $24,3 \pm 9,3$ & $25,2 \pm 9,1$ & - \\
ldade no diagnóstico (anos) & $14,0 \pm 7,5$ & - & \\
Duração da doença (anos) & $9,8 \pm 7,2$ & - & \\
Índice de massa corporal $\left(\mathrm{kg} / \mathrm{m}^{2}\right)$ & $22,3 \pm 3,4$ & $23,4 \pm 3,8$ & - \\
PAS (mmH) & $112,3 \pm 10,9$ & $110,2 \pm 10,8$ & 0,4550 \\
PAD (mmHg) & $70,2 \pm 10,6$ & $68,1 \pm 6,9$ & 1,3338 \\
\hline
\end{tabular}

$\mathrm{PAS}=$ pressão arterial sistólica; $\mathrm{PAD}=\mathrm{PA}$ diastólica. 
adição de brometo de potássio $(4,5 \mathrm{~g}$ de $\mathrm{KBr}$ a cada $9 \mathrm{~mL}$ de plasma) com posterior preparo dos tubos de ultracentrifugação com $20 \mathrm{~mL}$ de solução salina a $0,9 \%$ e os $9 \mathrm{~mL}$ de plasma anteriormente preparados. Estes tubos foram, então, centrifugados a $4{ }^{\circ} \mathrm{C}$ por 3 horas a 150.00xg. Após a ultracentrifugação, a banda de lipoproteínas com densidade entre 1,019 e $1,063 \mathrm{~g} / \mathrm{mL}$, compatível com a do LDL, foi coletada $(9,10)$. Este material teve sua concentração protéica dosada pelo método do Biureto (11) e ajustada para $0,2 \mathrm{mg} / \mathrm{mL}$. Ao material obtido foi adicionado sulfato de cobre $\left(\mathrm{CuSO}_{4}\right) 20 \mathrm{mM}$ na proporção de $1 \mu \mathrm{L}$ para cada $1 \mathrm{~mL}$ de LDL, e esta solução foi colocada em banho a $37^{\circ} \mathrm{C}$ por 24 horas.

A avaliação do LDL oxidado foi feita de forma indireta através do cálculo do coeficiente de oxidação desta partícula $(12,13)$, o qual utiliza em sua fórmua a absorvância em 3 comprimentos de onda da luz UV, a saber: $205 \mathrm{~nm}, 232 \mathrm{~nm}$ e $280 \mathrm{~nm}$.

Foram realizadas leituras no espectrofotômetro Schimadzu nos comprimentos de onda acima mencionados nos momentos pré adição e 1, 3, 6 e 24h após a adição do $\mathrm{CuSO}_{4}$, para avaliar a suscetibilidade do LDL ao processo oxidativo. O coeficiente de oxidação foi calculado através da seguinte fórmula: Abs 205 - Abs 280 / Abs 232 - Abs 280, onde Abs 205= leitura das duplas ligações dos ácidos graxos poli-insaturados dos fosfolipídeos do LDL; Abs 232= leitura dos dieno conjugados; e Abs $280=$ leitura da fração protéica do LDL.

\section{ANÁlISE ESTATísticA}

Os resultados de todas as variáveis analisadas foram apresentados como média e desvio padrão. Foi utilizado o teste $t$ Student não pareado para comparação das médias entre os dois grupos analisados. Para testar as correlações entre as variáveis de controle metabólico e a suscetibilidade do LDL à oxidação in vitro, utilizamos a correlação de Pearson.

\section{RESULTADOS}

Os resultados do controle glicêmico e do perfil lipídico estão na tabela 2. Observamos que o controle glicêmico dos pacientes estudados era regular e que não houve diferença estatisticamente significativa no perfil lipídico dos dois grupos avaliados.

Os pacientes com DMl apresentaram média de coeficiente de oxidação basal semelhante à dos sem DM. Entretanto, após a adição de sulfato de cobre, houve oxidação mais precoce nos pacientes com DM do que nos sem DM (tabela 3) (figura 1).

Houve diferença significativa na resposta do LDL dos sem DM e dos pacientes com DMl ao estímulo oxidativo 3 horas após a adição do sulfato de cobre. Ao analisarmos a interferência das variáveis de controle metabólico na suscetibilidade desta molécula à oxidação, encontramos correlação importante entre o Cox3h e a GPP e a HbAlc $(\mathrm{r}=-0,2511$; p $<0,05$ e $\mathrm{r}=$ $-0,2541 ; \mathrm{p}<0,05$, respectivamente) (figuras 2 e 3 ).

\section{DISCUSSĀO}

O presente estudo avaliou a suscetibilidade do LDL à oxidação in vitro em pacientes com DMl e indivíduos saudáveis pareados por sexo, idade e IMC. Houve análise também da interferência das variáveis de controle metabólico neste parâmetro.

Os dados na literatura são controversos no que diz respeito ao comportamento do LDL de pacientes com DMl frente ao stress oxidativo (14-16). Este conflito pode refletir diferença nas populações estudadas no que diz respeito ao grau de hiperglicemia, presença e severidade de complicações do DM, conteúdo de vitaminas na dieta e, possivelmente, raça (17). Os resultados obtidos na nossa amostra estão de

\begin{tabular}{|c|c|c|c|}
\hline Variáveis & DM1 & Sem DM & $\mathbf{p}$ \\
\hline $\begin{array}{l}\text { Glicemia de jejum (mg/dL) } \\
\text { Glicemia pós-prandial (mg/dL) } \\
\text { HbAlc (\%) } \\
\text { Indice de controle (\%) } \\
\text { Colesterol total (mg/dL) } \\
\text { HDL (mg/dL) } \\
\text { LDL (mg/dL) } \\
\text { Triglicerídeos (mg/dL) }\end{array}$ & $\begin{aligned} 182,0 & \pm 127,2 \\
226,5 & \pm 126,8 \\
8,3 & \pm 1,8 \\
1,34 & \pm 0,28 \\
172,1 & \pm 39,3 \\
47,1 & \pm 11,4 \\
111,8 & \pm 29,9 \\
78,1 & \pm 36,9\end{aligned}$ & $\begin{aligned} 80,4 & \pm 6,8 \\
96,6 & \pm 16,5 \\
4,5 & \pm 0,6 \\
0,72 & \pm 0,09 \\
189,9 & \pm 49,9 \\
46,3 & \pm 9,9 \\
122,5 & \pm 44,4 \\
96,0 & \pm 59,7\end{aligned}$ & $\begin{array}{l}0,1307 \\
0,7771 \\
0,3325 \\
0,3199\end{array}$ \\
\hline
\end{tabular}

$\mathrm{HDL}=$ lipoproteína de alta densidade; $\mathrm{LDL}=$ lipoproteína de baixa densidade. 
Tabela 3. Coeficientes de oxidação (Cox) do LDL do grupo de pacientes com DM1 e sem DM no momento da coleta dos exames.

\begin{tabular}{lccc}
\hline Cox & DM1 & Sem DM & p \\
\hline Basal & $9,7 \pm 1,9$ & $9,9 \pm 1,6$ & 0,7590 \\
Cox 1h & $8,8 \pm 1,9$ & $9,4 \pm 1,4$ & 0,1849 \\
Cox 3h & $6,7 \pm 1,5$ & $7,8 \pm 1,5$ & 0,0123 \\
Cox 6h & $5,3 \pm 1,3$ & $5,9 \pm 1,3$ & 0,0706 \\
Cox 24h & $4,1 \pm 1,2$ & $4,4 \pm 1,0$ & 0,3907 \\
\hline
\end{tabular}

Basal= coeficiente de oxidação basal (pré-adição de sulfato de cobre); Cox 1, 3, 6 e 24h= coeficiente de oxidação repectivamente 1, 3, 6 e 24 horas após adição de sulfato de cobre.

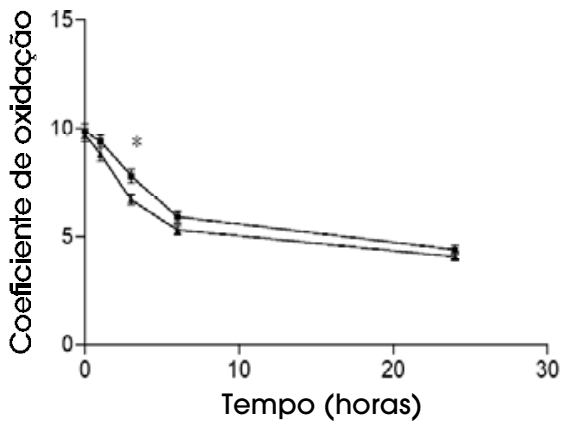

Figura 1. Curva de oxidação do LDL ${ }^{*} \mathrm{p}=0,0123$

acordo com os dados de Tsai e cols. (14), que também identificaram maior suscetibilidade do LDL à oxidação e correlação significativa com o controle glicêmico. Nosso trabalho traz, entretanto, um dado novo, que é a importância da glicemia pós-prandial neste fator também no DMl, o que havia sido descrito apenas no DM2.

Em nossa amostra, não houve diferença entre os dois grupos no momento pré-adição do agente oxidante, mostrando que, em estado pré-estímulo, não há diferença neste componente pró-aterogênico. Entretanto, 3 horas após a sua adição, o LDL dos pacientes com DMl apresentou-se mais oxidado. A partir de 6 horas após o início do estímulo oxidativo, ambos os grupos voltaram a ter a mesma resposta oxidativa.

Esta maior suscetibilidade nos pacientes com DMl não pode ser justificada pela presença de dislipidemia (18), então outros fatores são os determinantes desta situação.

No grupo estudado, encontramos correlação negativa do Cox3h com a GPP e a HbAlc, ou seja, quanto maiores os níveis destas variáveis menor o Cox3h. Isto pode ter relação com o fato do LDL glicosilado ser mais suscetível à oxidação (19), ou ainda com a capacidade que o estado hiperglicêmico tem de estimular a formação de radicais livres, o que causaria um maior consumo dos antioxidantes endógenos e permitiria que o LDL se tornasse mais suscetível à oxi-

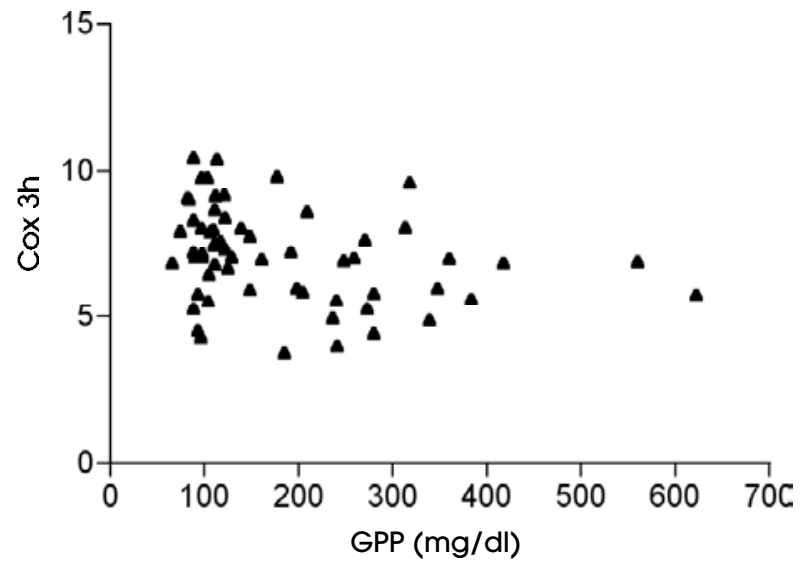

Figura 2. Correlação entre o Cox 3h e a glicemia pós-prandial $r=-0,2511 ; p<0,05$

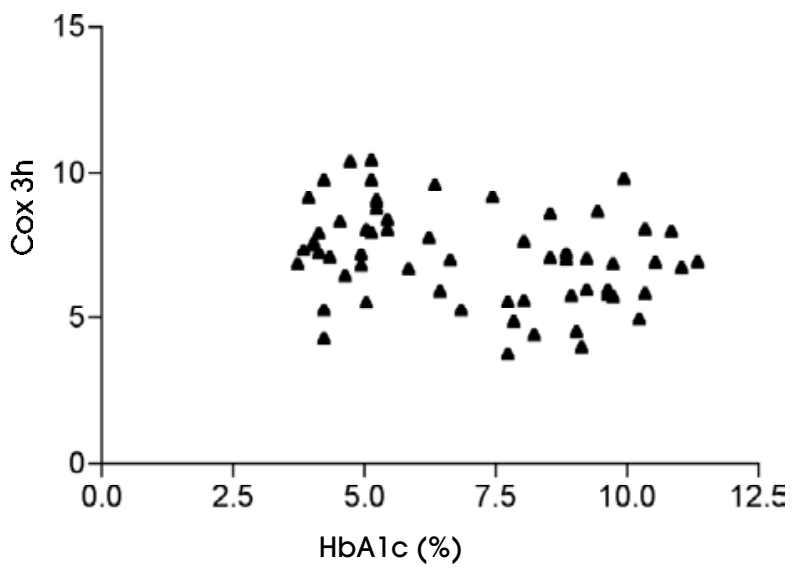

Figura 3. Correlação entre o Cox 3h e a HbAlc $r=-0,2541 ; p<0,05$

dação pelo sulfato de cobre. Existem dados que falam contra esta última possibilidade, como o conteúdo normal ou mesmo elevado de vitamina E encontrado em pacientes com DMl (20). Por outro lado, Hoeldtke (2l) mostrou em seu estudo que os pacientes portadores de DMl têm níveis séricos diminuídos de ácido úrico, um anti-oxidante endógeno.

Arq Bras Endocrinol Metab vol 48 n 4 Agosto 2004 
Uma vez que a resposta do LDL ao sulfato de cobre é semelhante àquela determinada pela presença de macrófagos ativados, é possível que o comportamento desta molécula frente ao estímulo oxidativo in vitro reproduza as alterações por ela sofridas in vivo (13). Assumindo esta hipótese como verdadeira, a maior suscetibilidade do LDL ao processo oxidativo pode ser um dos mecanismos fisiopatológicos envolvidos na maior prevalência de doenças ateroscleróticas em pacientes com DMl que na população em geral (22).

Em conclusão, nossos dados mostram que, durante a dinâmica de oxidação, o LDL de pacientes com DMl foi mais precocemente oxidado in vitro que o dos sem DM, e que este comportamento teve correlação significativa com a glicemia pós-prandial e a hemoglobina glicosilada. São necessários estudos prospectivos a fim de estabelecer todos os fatores preditores deste comportamento do LDL durante a exposição a substâncias ou situações com características oxidantes.

\section{REFERÊNCIAS}

1. Ruderman NB, Williamson JR, Brownlee M. Glucose and diabetic vascular disease. FASEB J 1992;6:2905-14.

2. The DCCT Research Group. The effect of intensive treatment of diabetes on the development and progression of long-term complications in insulin-dependent diabetes mellitus. N Engl J Med 1993;329:977-86.

3. Morrish NJ, Wang S-L, Stevens LK, Fuller JH, Keen H. The WHO Multinational Study Group: Mortality and causes of death in the WHO multinational study of vascular disease in diabetes. Diabetologia 2001;44(suppl. 2):S14-S21.

4. Droga G, Rich L, Stanton K, Watts GF. Endotheliumdependent and independent vasodilation studied at normoglycaemia in type 1 diabetes mellitus with and without microalbuminuria. Diabetologia 2001;44:593601.

5. Järvisalo MJ, Putto-Laurila A, Jartti L, Lehtimäki T, Solakivi T, Rönnemaa T, et al. Carotid artery intima-media thickness in children with type 1 diabetes. Diabetes 2002;51:493-8.

6. Chisolm GM, Steinberg D. The oxidative modification hypothesis of atherogenesis: an overview. Free Radical Biol Med 2000;28:1815-26.

7. Friedwald WT, Levy R, Fredrickson DS. Estimations of serum low density lipoprotein cholesterol without use of preparative ultracentrifuge. Clin Chem 1972;18:499502.

8. Chase HP, Jackson WE, Hoops SL, Cockerham RS, Archer PG, O'Brien D. Glucose control and the renal and retinal complications of insulin-dependent diabetes. JAMA 1989;261:1155-60.
9. Chung BH, Wilkinson T, Geer JC, Segrest JP. Preparative and quantitative isolation of plasma lipoproteins: rapid, single discontinuous density gradient ultracentrifugation in a vertical rotor. J Lipid Res 1980;21:284-91.

10. Heery JM, Kozak M, Stafforini DM, Jones DA, Zimmerman GA, Mclntyre TM. Oxidatively modified LDL contains phospholipids with platelet-activating factor-like activity and stimulates the growth of smooth muscle cells. J Clin Invest 1995;96:2322-30.

11. Gornall AG, Bardawill CJ, David MM. Determination of serum proteins by means of the Biuret reaction. J Biol Chem 1949;177:751-66.

12. Kim BS, LaBella FS. Comparison of analytical methods for monitoring autoxidation profiles of authentic lipids. J Lipid Res 1987;28:1110-7.

13. Esterbauer H, Gebicki J, Puhl H, Jürgens $G$. The role of lipid peroxidation and antioxidants in oxidative modification of LDL. Free Radical Biol Med 1992;13:341-90.

14. Tsai EC, Hirsch IB, Brunzell JD, Chait A. Reduced plasma peroxyl radical trapping capacity and increased susceptibility of LDL to oxidation in poorly controlled IDDM. Diabetes 1994;43:1010-4.

15. Liguori A, Abete P, Hayden JM, Cacciatore F, Rengo F, Ambrosio G, et al. Effect of glycaemic control and age on low-density lipoprotein susceptibility to oxidation in diabetes mellitus type 1. Eur Heart J 2001;22:2075-84.

16. Jenkins AJ, Klein RL, Chassereau CN, Hermayer KL, Lopes-Virella MF. LDL from patients with well-controlled IDDM is not more susceptible to in vitro oxidation. Diabetes 1996;45:762-7.

17. Kuyvenhoven JP, Meinders AE. Oxidative stress and diabetes mellitus. Pathogenesis of long-term complications. Eur J Int Med 1999; 10:9-19.

18. Orchard TJ. Dyslipoproteinemia and diabetes. Endocrinol Metab Clin North Am 1980;19:361-80.

19. Kobayashi K, Watanabe J, Umeda F, Nawata H. Glycation accelerates the oxidation of low-density lipoprotein by copper ions. Endocrinol J 1995;42:461-5.

20. Nhadimana J, Dorchy H, Vertongen F. Activité anti-oxydante erythrocytaire et plasmatique dans le diabète de type 1. Presse Méd 1996;25:188-92.

21. Hoeldtke RD, Bryner KD, McNeill DR, Hobbs GR, Riggs JE, Warehime SS, et al. Nitrosative stress, uric acid, and peripheral nerve function in early type 1 diabetes. Diabetes 2002;51:2817-25.

22. Krolewski AS, Warram JH, Valsania P, Martin BC, Laffel LM, Christlieb AR. Evolving natural history of coronary artery disease in diabetes mellitus. Am $\mathbf{J}$ Med 1991;90:S56-S61.

\section{Endereço para correspondência:}

Simone H. de Castro

Rua Maestro Vila Lobos 01, apto. 202

20260-220 Rio de Janeiro, RJ

e-mail: simone_hc@ig.com.br 